\title{
Beta-Rezeptorenblocker bei Patienten mit obstruktiven Atemwegserkrankungen - Risiken und Alternativen
}

H. Worth

\author{
Beta Receptor Blockers in Patients with Obstructive Respiratory Diseases - \\ Risks and Alternatives
}

\section{Zusammenfassung}

Wegen ihrer bronchokonstriktorischen Wirkung sollten BetaBlocker bei den obstruktiven Atemwegserkrankungen Asthma und COPD gemieden werden, wenn medikamentöse Alternativen bestehen. Dies ist für die arterielle Hypertonie, das Glaukom, supraventrikuläre und ventrikuläre Rhythmusstörungen der Fall. Indiziert ist der Einsatz Beta-1-selektiver Substanzen bei Patienten mit koronarer Herzkrankheit nach Herzinfarkt, da der Nutzen mit Reduktion von Morbidität und Mortalität durch die koronare Herzkrankheit die potenziellen Risiken bei sorgfältiger Beobachtung des Patienten und geringer Initialdosis des BetaBlockers überwiegt. Bei Patienten mit Asthma bronchiale sollten Beta-Blocker angesichts des gegenüber COPD-Patienten höheren Risikos des Auftretens einer schweren Atemwegsobstruktion prinzipiell nicht eingesetzt werden.

\section{Abstract}

The use of beta-blockers should be avoided in the treatment of patients with asthma and COPD because of their broncho-constrictive effect if there are medical alternatives available. This is the case in arterial hypertension, glaucoma, supraventricular and ventricular dysrhythmia. The use of beta-I-selective drugs is indicated in the treatment of patients with coronary heart disease after myocardial infarction, since the benefit of reduction of morbidity and mortality through the coronary heart disease outweights the potential risks if the patient is under close and careful observation and the initial dose of the beta-blocker is low. On principle patients suffering from asthma bronchiale should not be treated with beta-blockers because the risk of severe airway obstruction is higher than in patients with COPD.
Die Substanzklasse der Beta-Rezeptorenblocker wird in der Behandlung zahlreicher Erkrankungen erfolgreich eingesetzt (Tab.1). Besondere Bedeutung haben Beta-Rezeptorenblocker in der Pharmakotherapie von kardiovaskulären Erkrankungen wie der arteriellen Hypertonie, der koronaren Herzkrankheit, Herzrhythmusstörungen und der chronischen Herzinsuffizienz gewonnen. Der Wirkungsmechanismus der Beta-Blocker beruht darauf, die Bindung der Katecholamine an Beta-Rezeptoren zu hemmen. Die einzelnen Beta-Blocker entfalten prinzipiell ähnliche Wirkungen, Sie unterscheiden sich jedoch in einigen pharmakodynamischen und pharmakologischen Eigenschaften. Grundsätzlich lassen sich unselektive Blocker (Propanolol, Nado- lol, Carvedilol u.a.) von Beta-1-selektiven Rezeptorenblockern (Atenolol, Bisoprolol, Metoprolol, Nevibolol u.a.) unterscheiden. Trotz relativer Beta-1-Selektivität ist diese nicht strikt vorhanden - ein Aspekt, der besondere Bedeutung bei obstruktiven Atemwegserkrankungen erlangt. Ein weiteres Unterscheidungsmerkmal ist die intrinsisch-sympathomimetische Aktivität einiger Beta-Blocker (Pindolol, Oxprenolol u.a.).

Angesichts der positiven Effekte der Beta-Blocker auf die Morbidität und vor allem auch auf die Mortalität bei kardiovaskulären Erkrankungen einerseits und der bronchokonstriktorischen Wirkung andererseits ist der Einsatz dieser Substanzgruppe für Pa- 
Tab. 1 Wesentliche Indikationen für den Einsatz von Beta-Blockern

\section{arterielle Hypertonie}

koronare Herzkrankheit, insbesondere Zustand nach Myokardinfarkt

Herzrhythmusstörungen supraventrikulärer und ventrikulärer Genese

chronische Herzinsuffizienz

hyperkinetisches Herzsyndrom

Glaukom

Hyperthyreose

Migräne

Phäochromozytom

essenzieller Tremor

portale Hypertension
Allerdings stellen neuere Untersuchungsergebnisse wegen der gegenüber neueren Antihypertensiva wie Calcium-Antagonisten vom Dihydropyridin-Typ bzw. ACE-Hemmern geringeren Reduktion des Schlaganfallrisikos [9] sowie der häufigeren Auslösung eines Diabetes mellitus durch Beta-Blocker [2] den Stellenwert der Beta-Blocker in der Therapie der arteriellen Hypertonie infrage [9]. Alternativen zur Behandlung des Hochdrucks bei Patienten mit Asthma oder COPD sind Calcium-Antagonisten, Diuretika, AT-1-Antagonisten und mit Einschränkung auch ACE-Hemmer, die allerdings zu Husten führen und damit Patienten mit obstruktiven Atemwegserkrankungen zusätzlich beeinträchtigen können.

\section{Koronare Herzkrankheit}

Bei der chronischen koronaren Herzkrankheit ebenso wie bei akuten Koronarsyndromen senken Beta-Blocker den myokardialen Sauerstoffverbrauch und wirken daher antiischämisch. Bei der akuten Myokardischämie unter Einschluss des akuten Myokardinfarktes wird durch den konsequenten Einsatz von BetaBlockern die Mortalität wirksam gesenkt. Dabei spielt auch die Unterdrückung lebensbedrohlicher ventrikulärer Arrhythmien eine bedeutsame Rolle. Seit vielen Jahren ist der hohe Stellenwert von Beta-Rezeptoren-Blockern in der Sekundärprophylaxe nach Herzinfarkt nachgewiesen.

\section{Chronische Herzinsuffizienz}

Eine neue Indikation haben Beta-Rezeptorenblocker in der Therapie der chronischen Herzinsuffizienz gewonnen. Für die kardioselektiven Beta-Rezeptorenblockern Metoprolol, Bisoprolol und Nevibolol wurden eine verringerte Mortalität, eine Zunahme der linksventrikulären Pumpfunktion und eine verbesserte Herzleistung gezeigt. Die Empfehlung zum Einsatz von Beta-Rezeptorenblockern bei Herzinsuffizienz gilt nicht nur für ischämisch bedingte, sondern für alle Formen der systolischen Herzinsuffizienz.

\section{Unerwünschte Effekte durch Beta-Blocker bei obstruktiven} Atemwegserkrankungen

Die Verschlechterung eines vorbestehenden Asthma bronchiale unter Beta-Blockern wurde bereits kurz nach Einführung dieser Substanzklasse in die klinische Praxis beobachtet [10]. Obwohl diese Eigenschaft der Beta-Blocker seit langem bekannt ist, wird immer wieder über lebensbedrohliche Zwischenfälle, insbesondere bei Asthmatikern, auch bei Patienten mit leichtem Schweregrad der Erkrankung, unter Einsatz von Beta-Blockern berichtet [6]. Die zur Bronchokonstriktion führende Dosis des BetaBlockers kann insbesondere bei Asthmatikern in Abhängigkeit von den Begleitumständen (Infekte, Allergenexpositionen) niedrig sein. So wird selbst durch den Einsatz betablockerhaltiger Augentropfen in der Behandlung des Glaukoms über schwere Asthmaanfälle bis hin zu Todesfällen berichtet [19].

Pharmakologischer Hintergrund ist die Tatsache, dass eine Blockade betaadrenerger Rezeptoren, die am Vorhof- und Ventrikel-
Beta-Rezeptoren spielen eine zentrale Rolle in der Behandlung kardiovaskulärer Erkrankungen. Sie gehören nach aktuellen Leitlinien [3] zu den Medikamenten der 1. Wahl in der Therapie des Bluthochdrucks. 
myokard eine Hemmung adrenerg vermittelter positiv inotroper und chronotroper Effekte bewirkt, an der glatten Muskulatur, speziell der glatten Atemwegsmuskulatur, eine Konstriktion auslöst.

Während am Vorhofmyokard überwiegend Beta-2-adrenerge Rezeptoren vorhanden sind, liegen im Myokard der Ventrikel Beta-1- und Beta-2-adrenerge Rezeptoren vor, die glatte Atemwegsmuskulatur ist hingegen nur mit Beta-2-adrenerge Rezeptoren ausgestattet.

Heute werden neben unspezifischen Betarezeptorenblockern wie Carvedilol und Sotalol überwiegend kardioselektive Substanzen wie Metoprolol, Atenolol und Bisoprolol, neuerdings auch Nevibolol eingesetzt, die geringere unerwünschte Effekte auf die Beta-2-Adrenergenrezeptoren der Atemwegsmuskulatur aufweisen. Ferner wird die bronchodilatatorische Wirkung von Beta-2-Rezeptorantagonisten wie Salbutamol durch kardioselektive Beta-Rezeptorenblocker weniger als durch unselektive Substanzen gehemmt [4]. In hoher Dosis können jedoch auch kardioselektive Beta-Rezeptorenblocker ihre Beta-1-Selektivität verlieren [17].

Mehr als $90 \%$ der Asthmatiker zeigen nach Gabe des unselektiven Beta-Blockers Propranolol eine Bronchokonstriktion [18]. Das Ausmaß der Bronchokonstriktion bei Applikation eines Betablockers kann hierbei nicht sicher vorhergesagt werden und korreliert offensichtlich nicht mit dem Schweregrad einer bronchialen Hyperreaktivität. Die Sensitivität gegenüber Beta-Blockern ist ausgeprägter bei Patienten mit gutem Ansprechen auf Beta2-Sympathomimetika im Bronchospasmolyse-Test [7]. Dies wird gestützt durch die Bebachtung bei Patienten mit COPD, bei denen nach Gabe von Beta-Blockern seltener eine Verschlechterung der Lungenfunktion auftritt $[8,12]$. Ferner ist die Verschlechterung der Lungenfunktion meist geringer ausgeprägt bei Patienten mit COPD als bei Asthmatikern (cf 16).

Beta-Blocker mit intrinsischer Aktivität haben sich in der Behandlung nach Herzinfarkten und bei chronischer Herzinsuffizienz nicht bewährt, so dass ihr Einsatz auch bei Patienten mit obstruktiven Atemwegserkrankungen angesichts besserer Alternativpräparate nicht indiziert ist.

Um die Effekte kardioselektiver Beta-Blocker auf die Atmung bei Patienten mit COPD zu untersuchen, wurde von der Gruppe um Salpeter und Salpeter [15] eine Cochrane-Analyse durchgeführt, die 11 randomisierte, kontrollierte Studien mit Gabe von Einzeldosen und 8 Stunden mit länger dauernder Anwendung (2 Tage bis 12 Wochen) umfasste. Diese Metaanalyse zeigte im Vergleich zu Plazebo keinen signifikanten Effekt der Einmalgabe wie auch der längerdauernden Behandlung mit Beta-Blockern auf die $\mathrm{FEV}_{1}$. Die Schlussfolgerung der Autoren war, dass die Gabe kardioselektiver Beta-Blocker bei Patienten mit COPD keine signifikante Verschlechterung der Atemwegsfunktion und keine Zunahme der Inzidenz von Exazerbationen verursacht. Hierzu muss einschränkend festgehalten werden, dass die Zahl der eingeschlossenen Studien gering war und nur kurze Zeiträume betrachtet wurden. Außerdem liegen für unerwünschte Ereignisse wie Zunahme der Obstruktion bzw. Häufung von Exazerbationen oder Todesfällen unter Medikamenten meist keine randomisierten Studien vor, die in eine solche Metaanalyse Einlass finden.

Eine ähnliche Metaanalyse der gleichen Arbeitsgruppe wurde für Patienten mit „reversiblen“ bzw. „reaktiven“ Atemwegserkrankungen durchgeführt, d. h. für Patienten mit Anhaltspunkten für ein Asthma bronchiale $[13,14]$. Letzteres wurde jedoch nicht eindeutig definiert. Hierbei zeigte sich nach Einmalgabe kardioselektiver Betablocker eine Abnahme der $\mathrm{FEV}_{1}$ um 7,46\%, allerdings ohne Zunahme der Symptome. Unter länger dauernder Behandlung ( 3 Tage bis 4 Wochen) waren keine Unterschiede in der $\mathrm{FEV}_{1}$, der Symptomatik oder des Verbrauchs an Bronchodilatatoren nachweisbar $[13,14]$. Die Autoren folgern, dass die Gabe von Beta-Rezeptorenblockern auch bei Patienten mit leichter bis mittelschwerer reaktiver obstruktiver Atemwegserkrankung, also Asthmatikern, keine signifikanten unerwünschten Effekte auf die respiratorischen Funktion hätte und daher auch für diese Patienten nicht kontraindiziert sei. Diese Aussage muss kritisch gewertet werden, da in die Metaanalyse nur randomisierte, kontrollierte Studien eingingen und damit Berichte über Asthma-Todesfälle durch den Einsatz von Beta-Blockern nicht berücksichtigt wurden, zudem die Definition der reaktiven obstruktiven Atemwegserkrankungen unklar ist. Es ist vielfältig belegt, dass auch die Gabe kardioselektiver Beta-Rezeptorenblocker bei Asthmatikern mit hohem Risiko verbunden, und daher nicht indiziert ist (cf 19).

Bei Mischformen von Asthma und COPD besteht ebenfalls ein erhöhtes Risiko, so dass Beta-Rezeptorenblocker, wenn überhaupt, nur mit großer Vorsicht einzusetzen sind (cf 19).

Nebivolol als Beta-Rezeptorenblocker mit besonders hoher Beta1-Selektivität wird oft als sehr geeignet für den Einsatz bei Patienten mit obstruktiven Atemwegserkrankungen empfohlen. Diese Empfehlung beruht auf einer an 24 Patienten mit bronchialer Hyperreaktivität infolge Asthma, COPD oder unbekannter Ursache durchgeführten Untersuchung, die keine Veränderung der spirometrischen und ganzkörperplethysmographischen Kenngrößen der Obstruktion nach einmaliger Gabe von $5 \mathrm{mg}$ Nebivolol zeigte [11]. Die Schlussfolgerung, dass dieser Betablocker ebenso sicher wie Plazebo sei, erscheint verfrüht. Insbesondere konnte bisher nicht nachgewiesen werden, dass Nebivolol sicherer als andere kardioselektive Substanzen ist. Hierzu sind weitere, umfangreichere und vergleichende Studien notwendig.

Der prognostische Vorteil einer Therapie mit Betablockern bei Patienten mit COPD und Zustand nach Herzinfarkt ist durch Studien an großen Patientenkollektiven belegt [1,5]. Hierbei ist zu berücksichtigen, dass die COPD in der Studie von Gottlieb retrospektiv aus Krankenblättern und meist ohne Lungenfunktion diagnostiziert wurde, während Chen in einer Untersuchung an 54162 Patienten nach akutem Herzinfarkt zeigen konnte, dass die 1-Jahres-Mortalität bei Patienten mit leichter und mittelschwerer COPD durch eine Therapie mit Betablockern um $20-30 \%$ reduziert wurde, bei schwerer COPD hingegen keine signifikante Reduktion der Mortalität unter Betablockern zu beobachten war, wahrscheinlich deshalb, weil bei schwerer COPD diese limitierend für die Prognose der Patienten ist. 
zur Bekämpfung des hohen Blutdruckes e.V. http://www.uni-duesseldorf.de AWMF/II/II-ihypt.htm

Betablocker können bei Patienten mit Asthma bronchiale und COPD eine Bronchialobstruktion auslösen. Hiervon sind Asthmatiker häufiger, unkalkulierbarer und in stärkerem Ausmaß betroffen als Patienten mit COPD.

Zur Vermeidung einer durch Betablocker induzierten Bronchokonstriktion sollten bei obstruktiven Atemwegserkrankungen medikamentöse Alternativen bevorzugt werden, die für die meisten Indikationen, insbesondere die arterielle Hypertonie, das Glaukom, die supraventrikulären und meist auch ventrikulären Rhythmusstörungen zur Verfügung stehen. Für den Einsatz bei Betablocker bei chronischer Herzinsuffizienz und obstruktiven Atemwegserkrankungen liegen zuwenig Daten vor, um den Nutzen der Substanzgruppe bei gleichzeitig vorliegender COPD gegenüber den Risiken korrekt bewerten zu können. Nach Herzinfarkt bei erhöhtem kardiovaskulärem Risisko sind Beta-1-selektive Substanzen bei Patienten mit COPD indiziert, da der Nutzen mit Reduktion von Morbidität und Mortalität nach Infarkt die potenziellen Risiken bei sorgfältiger Beobachtung des Patienten und geringer Initialdosis des Betablockers überwiegt. Bei Patienten mit Asthma bronchiale sollten Betablocker angesichts des gegenüber COPD-Patienten höheren Risikos einer nicht kalkulierbaren schweren Atemwegsobstruktion prinzipiell nicht eingesetzt werden.

\section{Literatur}

${ }^{1}$ Chen J, Radford MJ, Wang Yet al. Effectiveness of beta-blocker therapy after acute myocardial infarction in elderley patients with chronic obstructive pulmonary disease or asthma. J Am Coll Cardiol 2001; 37 (7): $1950-1956$

2 Dahlöf B, Sever PS, Poulter NR et al. Prevention of cardiovascular events with an antihypertensive regimen of amlodipine adding perindopril as required, in the Anglo-Scandinavian Cardiac Outcomes TrialBlood-Pressure Lowering Arm (ASCOT-BPLA):a multicentre randomised controlled trial. Lancet 2005; 366: 895-906

${ }^{3}$ Deutsche Hochdruckliga. Leitlinien für die Prävention, Erkennung, Diagnostik und Therapie der arteriellen Hypertonie der Deutschen Liga
${ }^{4}$ Fogari R, Zoppi A, Tettamanti F et al. Comparative effects of celiprolol, propranolol, oxprenolol, and atenolol on respiratory function in hypertensive patients with chronic obstructive lung disease. Cardiovasc Drugs Ther 1990; 4 (4): 1145 - 1149

${ }^{5}$ Gottlieb SS, McCarter RJ, Vogel RA. Effect of beta-blockade on mortality among high-risk and low risk patients after myocardial infarction. N Engl J Med 1998; 339: 489-497

${ }^{6}$ Graft DF, Fowles J, McCoy CE et al. Detection of beta-blocker in people with asthma. Ann Allergy 1992; 69: 449-453

${ }^{7}$ Herwaarden CLA van. Betaadrenoceptor blockade and pulmonary function in patients suffering from chronic obstructive lung disease. J Cardiovasc Pharmacol 1983; 5: 46-50

${ }^{8}$ Lammers JWJ, Folgering HTM, Herwaarden CLA van. Ventilatory effects of long-term treatment with pindolol and metoprolol in hypertensive patients with chronic obstructive lung disease. $\mathrm{Br} \mathrm{J}$ Clin Pharmacol 1985; 20: 205-210

${ }^{9}$ Lindholm LH, Carlberg B, Samuelsson O. Should $\beta$ blockers remain first choice in the treatment of primary hypertension? A meta-analysis. Lancet 2005; 366: $1545-1553$

${ }^{10}$ MCNeill RS. Effect of beta-adrenergic blocking agent, propranolol, on asthmatics. Lancet 1964: $1101-1102$

${ }^{11}$ Matthys H, Giebelhaus V, Fallois J von. Nebivolol (Nebilet*) - ein sicherer Betablocker der dritten Generation - auch für Patienten mit obstruktiven Lungenerkrankungen? Z Kardiol 2001; 90 (10): 760 - 765

12 Perks W, Chatterjee S, Croxson R et al. Comparison of atenolol and oxprenolol in patients with angina or hypertension and coexistent chronic airways obstruction. Br J Clin Pharmacol 1985; 20: 205-210

${ }^{13}$ Salpeter S, Ormiston T, Salpeter E. Cardioselective beta-blockers for reversible airway disease. Cochrane Database 2002; Syst Rev 4: CD002992

${ }^{14}$ Salpeter SS, Ormiston T, Salpeter E. Cardioselective beta-blockers in patients with reactive airway disease: a meta-analysis. Ann Intern Med 2002; 137 (9): 715-725

${ }^{15}$ Salpeter SS, Ormiston T, Salpeter E et al. Cardioselective beta-blockers for chronic obstructive pulmonary disease. Cochrane Database 2002; Syst Rev 2: CD003566

${ }^{16}$ Tattersfield A. Respiratory function in the elderly and the effects of beta-blockade. Cardiovasc Drugs Ther 1990; 4: 1229-1232

17 Wellstein A, Palm D, Belz GG et al. Reduction of exercise tachycardia in man after propranolol, atenolol and bisoprolol in comparison to betaadrenoceptor occupancy. EUR Heart J 1987; 8 (Suppl M): 3-8

18 Woolcoock AJ, Anderson SA, Reat JK et al. Characteristics of bronchial hyperresponsiveness in chronic obstructive pulmonary disease and in asthma. Am Rev Respir Dis 1991; 143: 1438-1443

19 Worth H. Beta-Blocker bei Asthma und COPD - ein therapeutisches Dilemma? Pneumologie 2001; 55 (2): 53-56 